\title{
Interactive Selection of Optimal Fenestration Materials for Schematic Architectural Daylighting Design
}

\author{
Barbara Cutler a,*, Yu Sheng ${ }^{a}$, Steven Martin ${ }^{\mathrm{a}}$, Daniel Glaser ${ }^{\mathrm{b}}$, \\ Marilyne Andersen ${ }^{\mathrm{c}}$ \\ ${ }^{a}$ Computer Science Department, MRC 309A, \\ Rensselaer Polytechnic Institute, 110 8th Street, Troy, NY 12180-3590, USA \\ ${ }^{\mathrm{b}}$ Department of Language, Literature, and Communication, \\ Rensselaer Polytechnic Institute, 110 8th Street, Troy, NY 12180-3590, USA \\ ${ }^{\mathrm{c}}$ Building Technology Program, Department of Architecture, \\ Massachusetts Institute of Technology, Room 5-418, \\ 77 Massachusetts Avenue, Cambridge, MA 02139-4307, USA
}

\begin{abstract}
Complex fenestration systems, such as prismatic and laser cut panels, are emerging as attractive options in architectural design thanks to their high potential to assist in energy and comfort issues. These systems can be used to redirect intense illumination from the sun but have complex transmissive properties that in turn depend on continuously changing illumination conditions due to the dynamic nature of natural light. The resulting non-intuitive interactions with the built environment make it necessary to develop tools that adequately represent these systems' behavior to the architect. The method presented in this paper enables simulation of the direct and indirect illumination from the sun and sky throughout each day for different months of the year. The user can interactively explore the high-dimensional configuration space to select optimal materials early in the design process, using both qualitative and quantitative design criteria to make adjustments.
\end{abstract}

Key words: Global illumination, Radiosity, Bidirectional Transmission Distribution Function (BTDF), Architectural design, Daylighting simulation, Shadow volumes, Complex Fenestration Systems (CFS).

\footnotetext{
* Corresponding author. Tel: +1-518-276-3274; fax: +1-518-276-2529.

Email address: cutler@cs.rpi.edu (Barbara Cutler).

URL: http://www.cs.rpi.edu/ ${ }^{\sim}$ cutler/ (Barbara Cutler).
} 


\section{Introduction}

Designing with daylight shows great promise for reducing energy demands of buildings, increasing occupant comfort and well-being, and shaping delightful spaces $[1,2,3,4,5]$. There has been a resurgence of interest in using environmentally sustainable methods that take into account diurnal, seasonal, latitudinal, and climatic variables and the last few decades have seen an emergence of complex fenestration system (CFS) technologies to respond more efficiently to these parameters $[6,7,8,9]$.

But only if daylighting is accounted for early in the design process, when scale, appearance, and adjacencies of an evolving design are explored and critiqued, can it have a significant positive impact on the sustainability of the building. To assist the designer in accounting for the many factors that influence daylight during this crucial schematic design phase, computer simulations and/or studies with physical scale models can be very effective tools and provide useful and quick feedback. An architect may choose to study the interrelations among light, materials, and space in high-quality renderings or photographs whereas an engineer may analyze point-by-point light measurements. The investigation of many scenarios and conditions will be necessary in either case before conclusions can be reached regarding the most successful design choices.

Available tools cover a wide range in the level of complexity and accuracy of information that can be obtained. Traditional sunlight penetration analysis tools such as the heliodon are still taught and used during schematic design $[10,11,12]$. A physical mock-up of the design is placed on the heliodon table to provide instantaneous and intuitive qualitative feedback on direct sun penetration as the model is rotated and manipulated (Figure 1).

More advanced approaches include the diffuse sky component (daylight) in
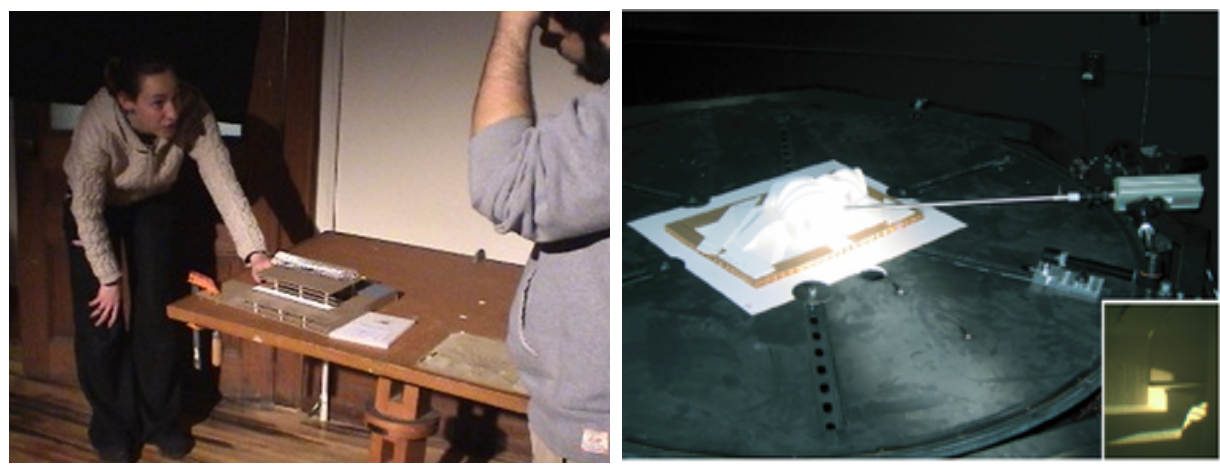

Fig. 1. The depth of direct beam light penetration in a model is easily ascertained with the heliodon. Interior views (right inset) may require endoscopic lenses depending on the model size. The heliodon table rotates along many dimensions relative to a fixed light source (the sun), facilitating exploration. 

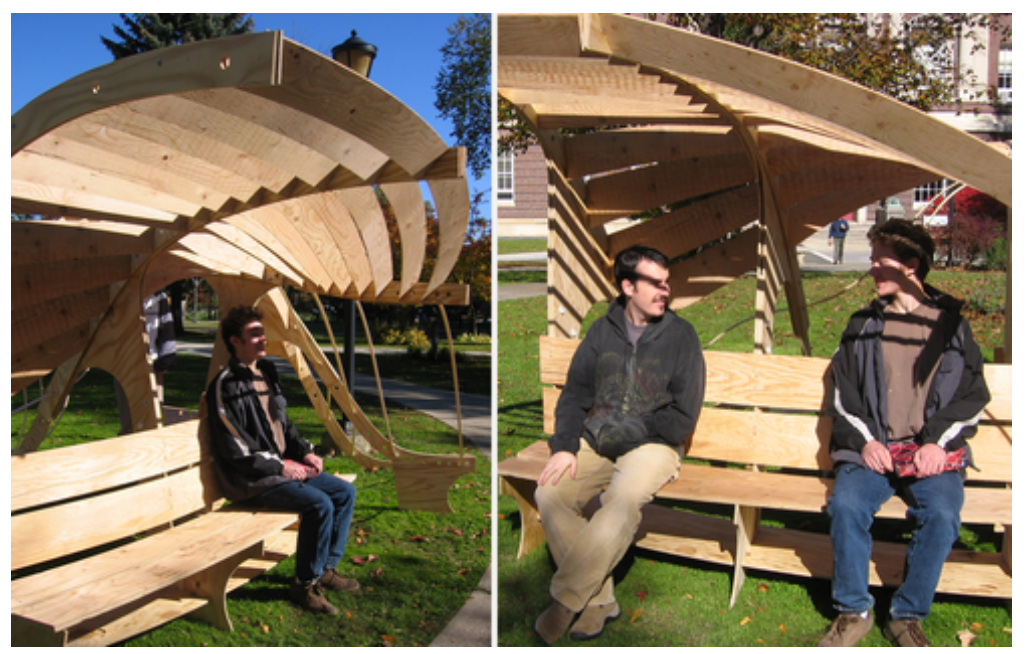

Fig. 2. Sunlight penetrates the louvres of this architectural studio project in an unanticipated way, causing discomfort. An interactive daylight renderer could have predicted this problem, allowing the designer to optimize the orientation of the louvres.

their analysis as well, either relying on artificial skies (mirror boxes, domes, scanning methods) for physical models $[13,14,12]$ or on virtual sky representations $[15,16]$.

The heliodon's equivalents in computer simulation are the daily and annual sun-path shadow analysis tools available in software like Sketchup [17] or Ecotect [18]. An illustrative example of a design that did not benefit from a careful sunlight penetration analysis is given in Figure 2, whose elegant assembly of curved elements unfortunately allows serious glare issues that could have been prevented with an appropriate preliminary study.

Physically-accurate computer simulations of global illumination, including radiosity and Monte Carlo ray tracing are available in some CAD programs; however, these lighting tools have not made significant inroads into the architecture community for early stage schematic design [19]. Furthermore, most architects are untrained or unaware of how to prepare their models or tune parameters for speedy yet accurate renderings. Thus, daylighting simulation software is very seldom used in academic or professional practice to inform design.

The renewed interest and innovation in fenestration products and designs heralded by recent environmental concerns add a new challenge to a smooth and successful design process. Their complex interactions with light make it tremendously difficult to predict what illumination effects will be generated depending on the sun position and sky conditions. Prismatic panels are an example of one such CFS and can be used to redirect illumination deep into the room if the facet orientations are chosen appropriately (Figure 3). These panels were popular in the 1890's; however, they fell out of use as electric 


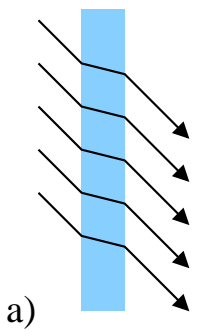

b)
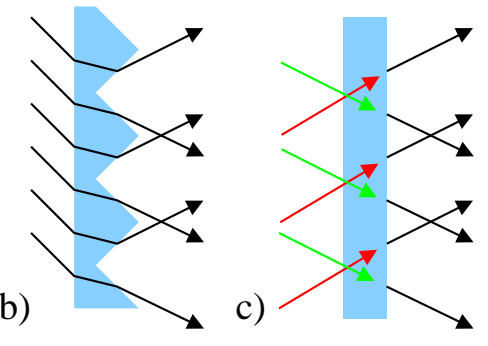

Fig. 3. a) In a flat pane of glass, light rays are refracted, but exit parallel to the input rays. b) Light rays that pass through a prismatic panel are refracted differently and exit in two different directions due to the microfaceting. c) We can reverse engineer the directions of two "fake suns" allowing us to render the specular refraction in real time (Section 3.4).

lighting became available in the early 20th century. The somewhat counterintuitive behavior of light refraction through these panels can cause confusion and architects are "not certain how to incorporate the prismatic glass into the aesthetic of a building" [20]. Furthermore, studies of occupants in existing buildings with advanced fenestration products reveal additional challenges for adoption: "This system produces not only uneven lighting on wall and working surfaces but also (unexpected, to the client) sharp shadows on the ceiling" [21].

Goniophotometers can be used to measure the Bidirectional Transmission Distribution Function (BTDF) of each fenestration product [22], making it possible to accurately predict (both qualitatively and quantitatively) the behavior of each material in a given building scenario. However, linking material characteristics to space illumination conditions is a difficult task because of the many variables involved and appropriate visualization tools are needed.

The method described in this paper focuses on efficiently predicting daylighting illumination and using 2D configuration space plots to evaluate the current design and optimize material selection early in the design process. It is illustrated in Figure 4 and includes:

- An interactive global illumination system for natural daylighting that is appropriate to the models used in schematic design.

- Combines computation of direct illumination (shadow volumes) with indirect illumination (radiosity) in a hybrid system.

- Handles non-diffuse BTDF for a variety of specular fenestration materials.

- Interactive plotting of 2D slices through the configuration space, allowing exploration of the optimization problem (rather than returning a single solution from an offline optimization).

- Demonstration of our system on several case study designs from our architectural collaborators. 
a)

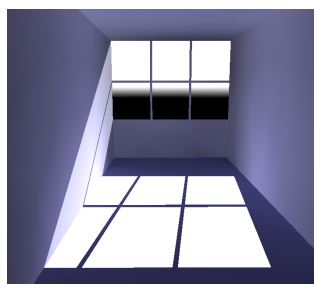

c)

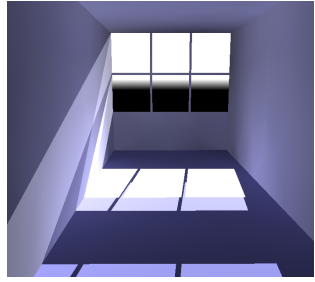

e)

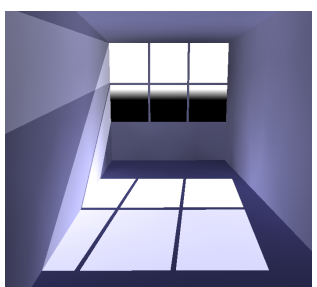

g)

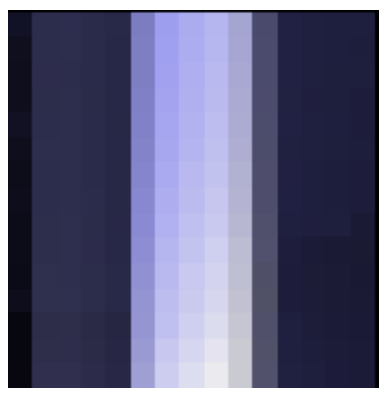

b)

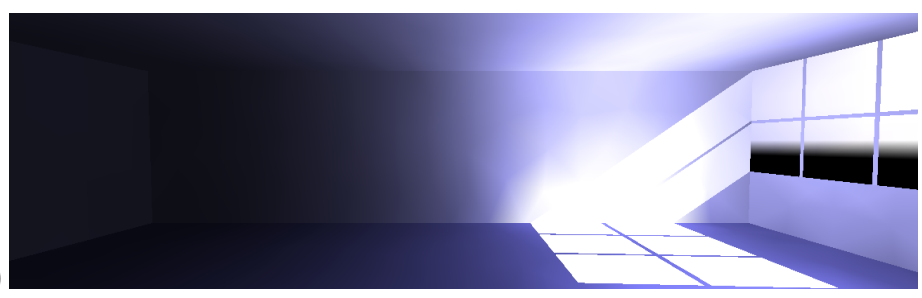

d)

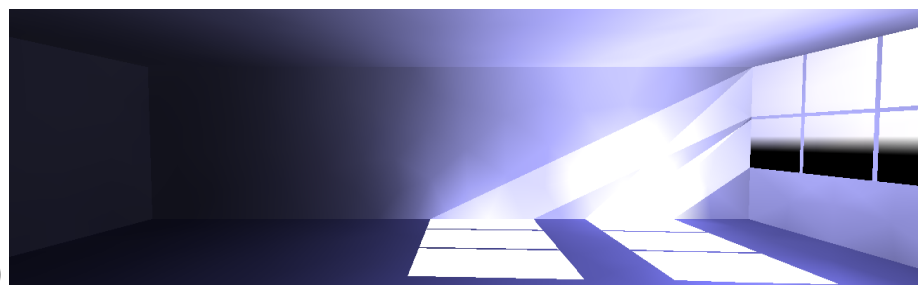

f)

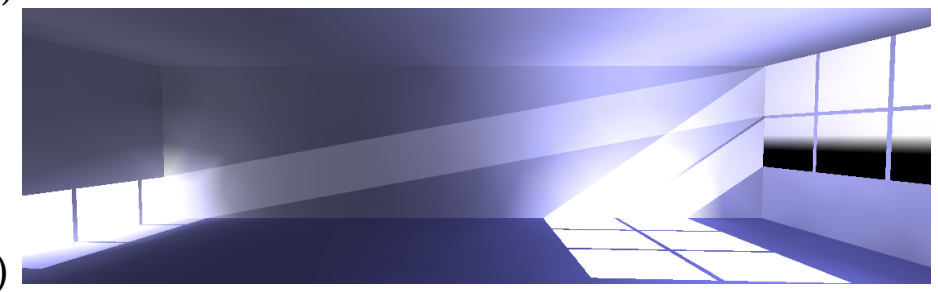

h)

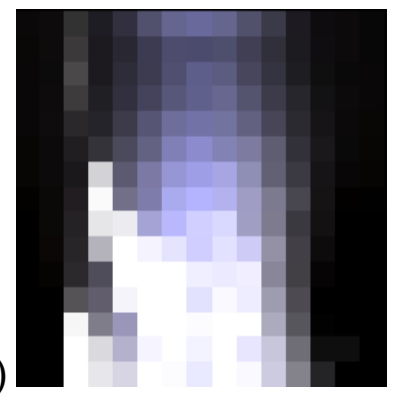

Fig. 4. Initially all six windows in this simple room scene are filled with a\&b) simple planar glass. Note how the far back corner of the room is dark. In c\&d) we replace the top 3 panes with a prismatic panel having shallow angles and in e\&f) we select a more extreme angle for the upper microfacets in these panels, which sends direct light to the far wall to more uniformly illuminate the room. To optimize the material selection for the center point of the floor at a given time and day of the year, we g) vary the two angular parameters of the prismatic material. Fixing the material, we $h$ ) vary the time of day and day of year and calculate the illumination on the center point of the floor.

\section{Related Work}

\subsection{Architectural Lighting Design Software}

The field of lighting design software is highly fractured, encompassing an enormous number of programs that are either commercially available or can be downloaded for free. A comprehensive survey [19] of these programs reported that more than $50 \%$ of the cited tools were based on the Radiance simulation 
engine [23, 24, 25]. Unfortunately, most architects do not use this tool frequently because model preparation is non-trivial and appropriate renderings can take from minutes to hours to create. Only expert users seem to have the necessary knowledge about the underlying algorithms to correctly adjust the numerous parameters to produce quick yet accurate and useful images and data. Front-end interfaces such as the "Radiance Control Panel" from Ecotect [18] have partly solved this problem but they generally only offer limited capabilities compared to Radiance.

Ecotect is a recent tool gaining popularity within the building technology community and is making its way into architectural design programs at the university level and in architectural practice. Within Ecotect one can perform lighting calculations with diffuse skies, optimize the shape of exterior shading devices, and perform other environmental evaluations such as thermal and energy analyses. This tool requires some training time and designs must be precisely annotated before analysis and simulation can begin.

A large range of interactive, easy-to-learn tools with a specific focus on early design stages are also found, such as the MIT Design Advisor [26], DIALEurope [27], and Daylight1-2-3 [28]. The simulations are limited to simple geometries and, except for Daylight1-2-3, rely on strong simplifying assumptions about the sky model. One of their major drawbacks is that they only provide quantitative information and have very limited visualization capabilities. Not being able to interactively view the illuminated space and quickly explore alternatives with both visual and performance criteria in mind seem to be one of the main reasons why computer simulations of global illumination are rarely used in the early stages of design.

\subsection{Computer Graphics Research on Global Illumination Simulations}

A popular method for architectural lighting simulation is radiosity, in which pairwise form factors describing the geometric relationships between surfaces are computed and used to distribute light between surfaces [29, 30, 31, 32, 33]. Alternative methods to radiosity exist for modeling indirect lighting such as irradiance caching, which progressively samples and interpolates scene irradiance values $[34,35,36]$. A more complete summary of recent advances for both interactive and offline global illumination can be found in [37].

Radiosity and irradiance caching are primarily useful for low-frequency elements of scene illumination. While a solution combining radiosity with ray tracing to obtain high-frequency lighting effects such as hard shadows has been described [38] and implemented with shadow mapping [39, 40], we are

not aware of other proposals that use our hybrid method of shadow volumes 
for per-pixel hard shadows of direct sun illumination and path-based radiosity for sky and indirect illumination.

Modern graphics hardware (the GPU) has been used to accelerate global illumination to interactive rates, including: intersection of rays against scene geometry [41]; precomputed light paths for static geometry [42]; GPU-assisted implementation of the hemicube form factor method [43]; and GPU implementation of irradiance and radiance caching [39]. Real-time ( $\geq 30$ frames per second) global illumination rendering of dynamic environments have been achieved using a coarse volumetric sampling grid [44]; however, the potential artifacts from shadow approximations may not yield the accuracy required for architectural applications. Recently, a new method called Antiradiance, which replaces the need for visibility computation has been demonstrated on the GPU to interactively (at 5-10 frames per second) update indirect illumination with moving characters and lights [40].

\subsection{Interactive, Human-Guided Optimization}

The process of architectural design can be naively characterized as a common optimization problem: Find the geometry from the space of all possible building designs that maximizes functionality and aesthetic for minimum cost. Unfortunately, many terms in this cost function cannot be easily quantified and the coefficients for each term are design specific and not known a priori. Even if we could specifically define a cost function, solving this discontinuous and high-dimensional non-linear optimization would be impractical. We are inspired by the Design Galleries [45] and Human-Guided Simple Search [46] approaches, which adequately sample the configuration space and leverage the user's intuition to navigate the space and avoid local minima. The interactive nature of these systems builds user confidence in the final solution.

\section{Our System}

In the following sections we outline the features of our rendering algorithm for schematic architectural daylighting design. Together they efficiently and accurately model the illumination and allow a designer to analyze, evaluate, and optimize his/her design. 


\subsection{Sampling the Direct Sun \& Sky Illumination}

Natural illumination in the built environment is provided not only from direct parallel rays of sunlight, but also by omni-directional illumination from the non-uniform, seasonally-varying sky hemisphere. We model the hemispherical distribution and relative intensity of the sky using standard models $[15,16]$.

We use forward ray tracing to cast rays from the sun and sky through windows in the model to compute the direct illumination for each surface. For the sun, we cast approximately 70,000 total parallel rays through all windows whose normal faces the sun position. For the sky, we choose approximately 5,000 samples on the hemispherical sky and approximately 50 total samples on all the windows and trace all rays starting from each point on the sky through each point on the windows. The samples are appropriately normalized by area. The direct illumination rays from the sun and sky are traced into the scene and the light is stored with the surface. We make note of which rays came from parallel sunlight rays versus the omni-directional skylight (this information is used in Section 3.3). For a typical relighting event, we cast roughly 320,000 rays through the scene. We use a spatial data structure to ensure that reasonable performance is achieved despite the large number of rays.

\subsection{Radiosity for Diffuse Reflections}

Light sampled from direct sun and sky illumination is distributed via diffuse reflection between surfaces using radiosity. First, each surface in the scene is partitioned into rectangular or triangular patches. Then, the classical radiosity equation[29] specifies the ideal diffuse light transportation between patches:

$$
B_{j}=E_{j}+\rho_{j} \sum_{i=1}^{N} B_{i} F_{j i}, \text { for } j=1, \ldots, N
$$

Here $B_{j}$ is the radiosity for each patch in the scene, $E_{j}$ is the self-emissive light intensity of patch $j, \rho_{j}$ is the reflectivity of patch $j$, and $F_{j i}$ is the form factor which represents the fraction of energy leaving $j$ and arriving directly at $i$. This linear equation can be solved using a Gauss-Seidel iterative method. The first two iterations are listed in the following equations:

$$
\begin{aligned}
& B_{j}^{(0)}=E_{j}^{(0)} \\
& B_{j}^{(1)}=E_{j}^{(1)}+\rho_{j} \sum_{i=1}^{N} B_{i}^{(0)} F_{j i}
\end{aligned}
$$


The first equation shows that the initial value of each patch is the self-emissive intensity. The second equation computes the illumination each patch receives directly from the light sources in the scene. At each patch, three scalar quantities are maintained: the direct illumination received from the sun and sky, the indirect illumination received on the face in the previous iteration, and the cumulative illumination reflected from the face.

\subsection{Factoring Direct and Indirect Illumination}

In most architectural scenes involving daylighting, light transfer due to diffuse reflection from surfaces dominates the indirect lighting. Additionally, hardedged shadows from the direct sun provide important visual cues that are necessary to understand the aesthetic of the space. Furthermore, the possibility of glare due to high contrast in the illumination values at the direct shadow boundaries must be considered. Per-pixel hard shadows greatly improve the perceived visual quality, but are usually not critical for computing accurate indirect illumination in diffuse-dominant scenes.

With a low-resolution mesh, traditional radiosity is likely to generate unacceptable visual artifacts from the direct illumination, as shown in Figure 5a. Traditionally, these artifacts are reduced by either significantly increasing the mesh resolution or employing discontinuity meshing [47]. However, this will also dramatically increase the computation time and is typically applied only for static lighting conditions.

In our method, we reduce these artifacts by factoring the radiosity solution into direct illumination (Figure 5b) and indirect illumination (Figure 5c) and replace the direct illumination by a fast per-pixel rendering method, called shadow volumes [48]. In our hybrid radiosity/shadow volumes technique, the radiosity is computed on a coarse per-face basis, while the shadows from direct sunlight are computed at render time on a per-pixel basis (Figure 5d). Although the sky contributes significantly to direct illumination, because it produces only soft shadows it is well represented in the original radiosity solution.

The direct illumination from the sun is generated with per-raster-sample computations using multi-pass stencil shadow volumes $[48,49,50]$. This algorithm is chosen because it is supported by almost all graphics hardware, and can achieve real-time speed with a complexity of $O(E)$, where $E$ is the number of window edges plus the number of silhouette edges for a given light direction [51]. To produce the final composite solution, the scene is first rendered using the direct sky illumination combined with total indirect illumination. Next, shadow volume polygons are generated as projections from the window 

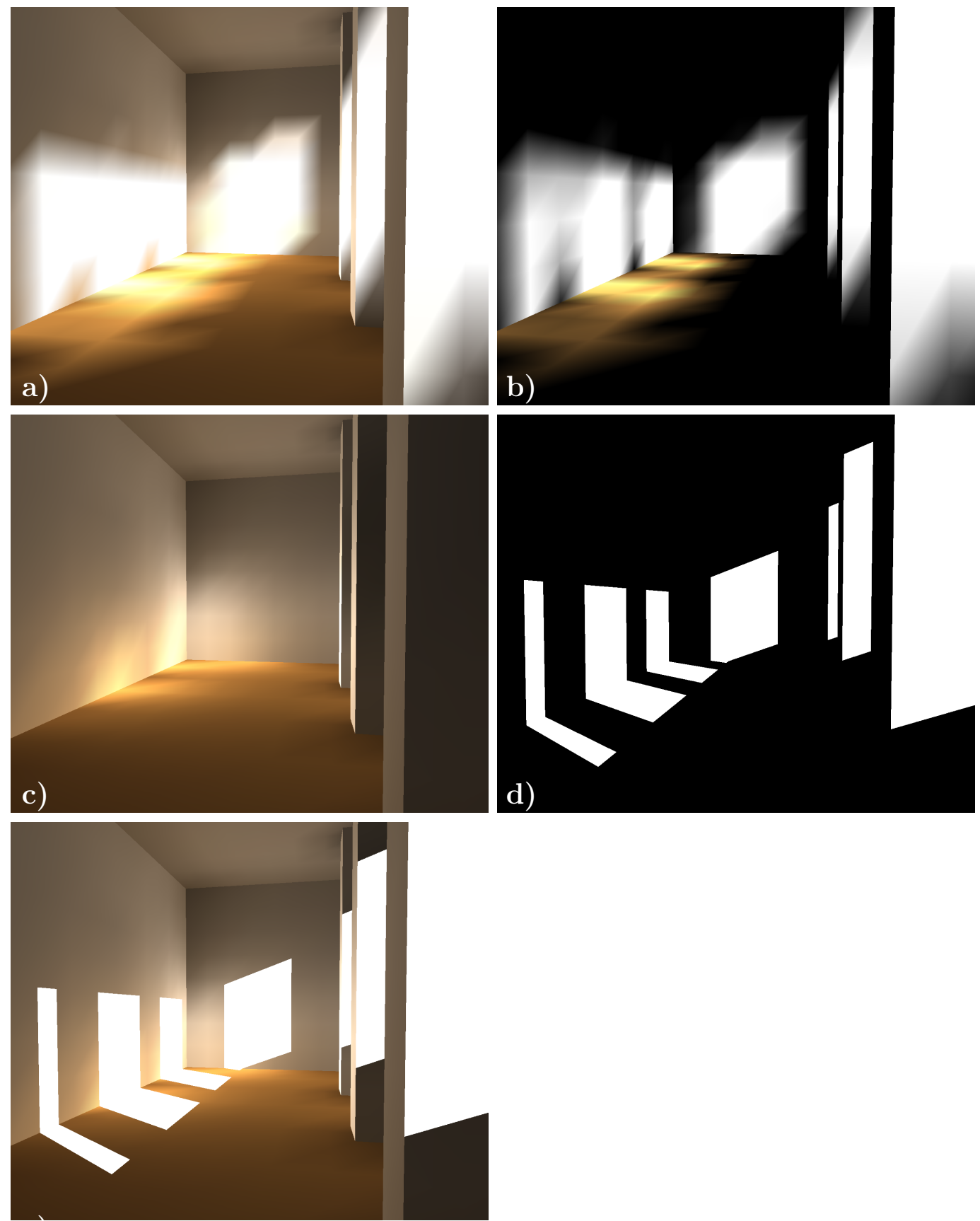

Fig. 5. The a) classical radiosity solution does not capture hard-edged shadows. We factor the radiosity solution into b) the first bounce direct illumination and c) the indirect illumination by subtracting b) from a). d) Shadow volumes are used to generate per-pixel hard shadows. e) Our hybrid radiosity/shadow volumes rendering is generated by adding c) and $d$ ). 

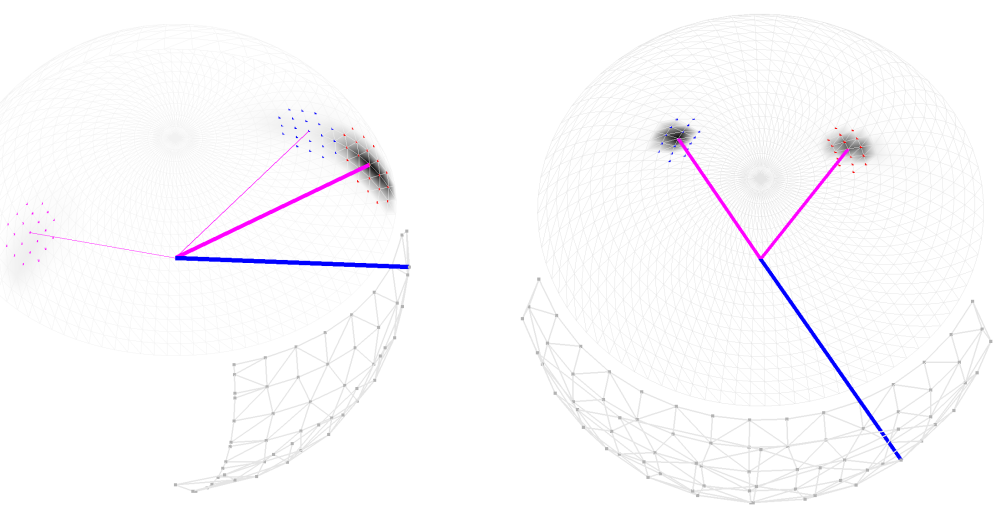

Fig. 6. Plots of the BTDF data for the laser cut panel CFS for two sample incoming light directions (shown in blue). Up to three lobes are fit to the data to model the specular outgoing illuminance (shown with different widths).

and silhouette edges and rendered to the stencil buffer. Finally, the per-pixel direct illumination from the sun is additively rendered to the frame modulated by the contents of the stencil buffer.

\subsection{Modeling the Specular Lobes of BTDF data}

We extend the method described in the previous section to model CFS such as prismatic and laser cut panels. These materials can be modeled as a set of one or more specular lobes. When the exact microfacet geometry of the material is known - for example, the prismatic panel shown in Figure 3 - we compute the orientation and relative intensity of the two lobes by tracing a recursive refractive ray through each of the microfacet orientations.

For other materials (such as the laser cut panels shown in Figure 6), we use the 4D BTDF measurement data produced at EPFL, Switzerland [52]. For each measured incoming light direction $\left(\theta_{i}, \phi_{i}\right)$ we greedily select $k$ outgoing directional lobes subtending $\alpha$ degrees that minimize the un-represented transmissive illuminance. For the laser cut panel dataset, we found that $k=3$ lobes of width $\alpha=23$ degrees were sufficient to represent $82-100 \%$ of the transmitted outgoing illuminance (Figure 6). When rendering illumination from an arbitrary incoming direction, we locate the three nearest measured incoming directions $\left(\theta_{i 0}, \phi_{i 0}\right),\left(\theta_{i 1}, \phi_{i 1}\right)$, and $\left(\theta_{i 2}, \phi_{i 2}\right)$ using a Delauney triangulation. Then we rank and correspond the lobes between each measured direction by decreasing intensity. Finally, the lobe direction and brightness is linearly interpolated for the queried direction. We have found this simple correspondence method to be sufficient for interpolation of a variety of specular BTDF data. However, we anticipate that this simplistic method may result in incorrect correspondences for some BTDF measurements and plan to implement a more robust correspondence technique in future work. 


\subsection{Rendering Complex Fenestration Systems}

Once the lobe directions and intensities are computed, we systematically render each specular reflection from the sun through the different fenestration materials. As before, we first render the direct sky illumination and total indirect illumination from radiosity. Then we loop through the distinct BTDF materials in the model, and for each of the $k$ specular lobes we position a fake sun such that non-refracting rays from the fake sun will be parallel to the outgoing specular lobe (Figure 3c) and scale the brightness of the fake sun by the transmittance value. Then we render the stencil buffer shadow volume for the window edges of that material. The number of rendering passes required for a scene with multiple complex fenestration materials is thus $1+2 * k * d$ where $d$ is the number of distinct fenestration materials and installation orientations. Figures 7 and 8 demonstrate our rendering method for a variety of CFS. For CFS that include a significant diffuse component, the diffuse radiosity emittance for those window patches can be set appropriately.

\subsection{Interactive Relighting}

To facilitate schematic architectural design that incorporates, responds to, and embraces daylighting, our system must support efficient, interactive recomputation of illumination when the sun is moved and the sky illumination varies with the time of day, season, and climate. We use progressive radiosity [30] to smoothly interpolate the illumination values as the lighting solution for the new sun and sky direct illumination is computed. When the sun is moved, we first recompute the direct light, and then redistribute the light. On each iteration of the "light shooting" radiosity solution, the updated incremental illumination for the old position is replaced by that of the current light position. By separating the direct sun illumination from direct sky illumination and indirect illumination, the system can provide real-time updates of the per-pixel hard shadows as the sun moves. Meanwhile, a separate thread of computation re-calculates at interactive rates the radiosity global illumination data for each face in the mesh. Figure 12 and the companion videos available on our website show screen captures of interactive relighting within our system.

\subsection{D Configuration Space Plots for Optimization}

The interactive exploration and selection of CFS with optimal performance is possible within our rendering system. The user fixes certain variables while allowing others to vary in order to slice through the high-dimensional configuration space. The user positions one or more rectangles representing target 

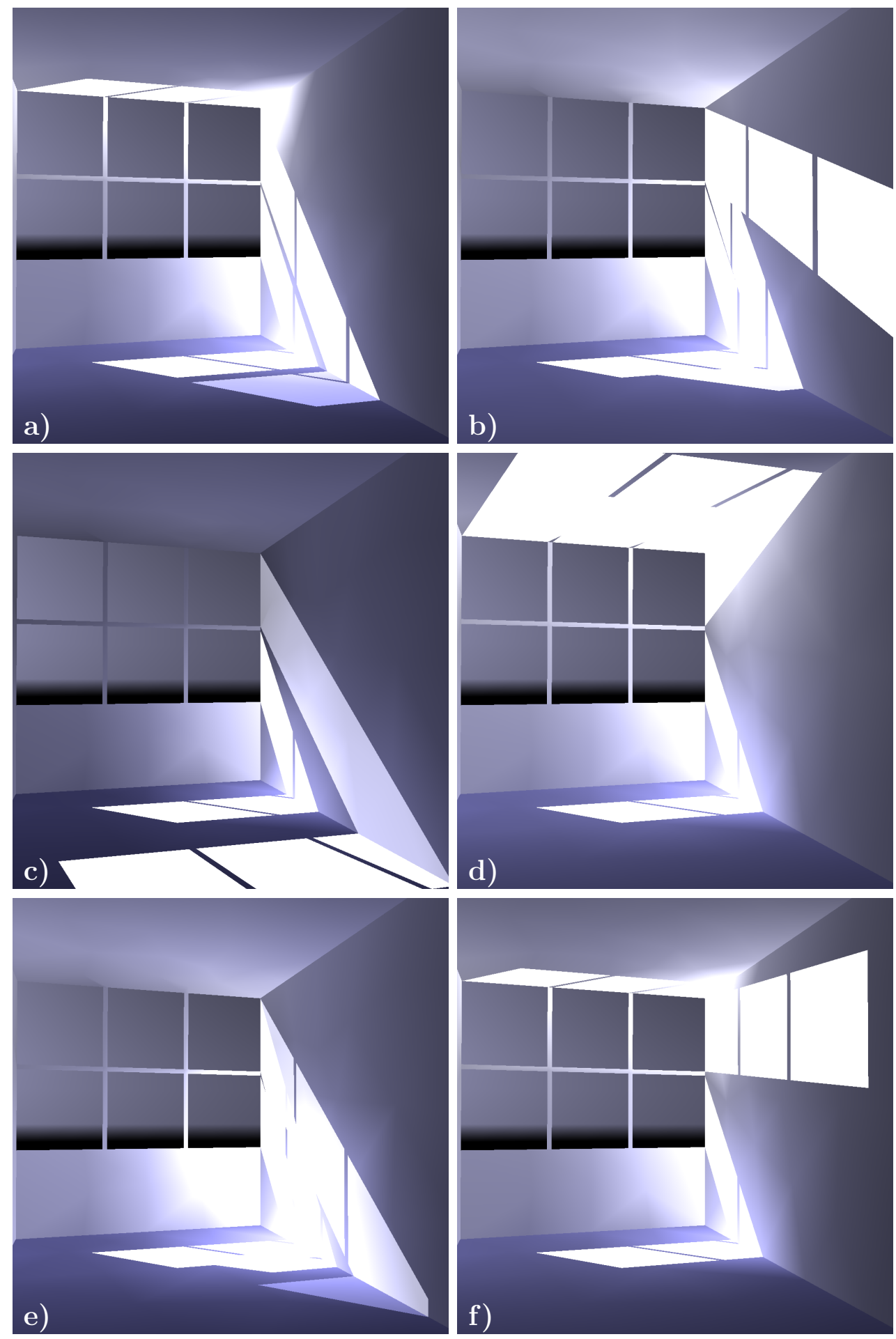

Fig. 7. Sample CFS renderings in a small test room with south facing windows at $10 \mathrm{am}$ on March 21st. The bottom 3 window panes are plain glass in all images. The top 3 panes are: a) laser cut panel, b) optical film (interior), c) perforated blind (open), d) Lumitop ${ }^{\mathrm{TM}}$, e) mirrored Venetian blind, and f) Serraglaze $^{\mathrm{TM}}$. 

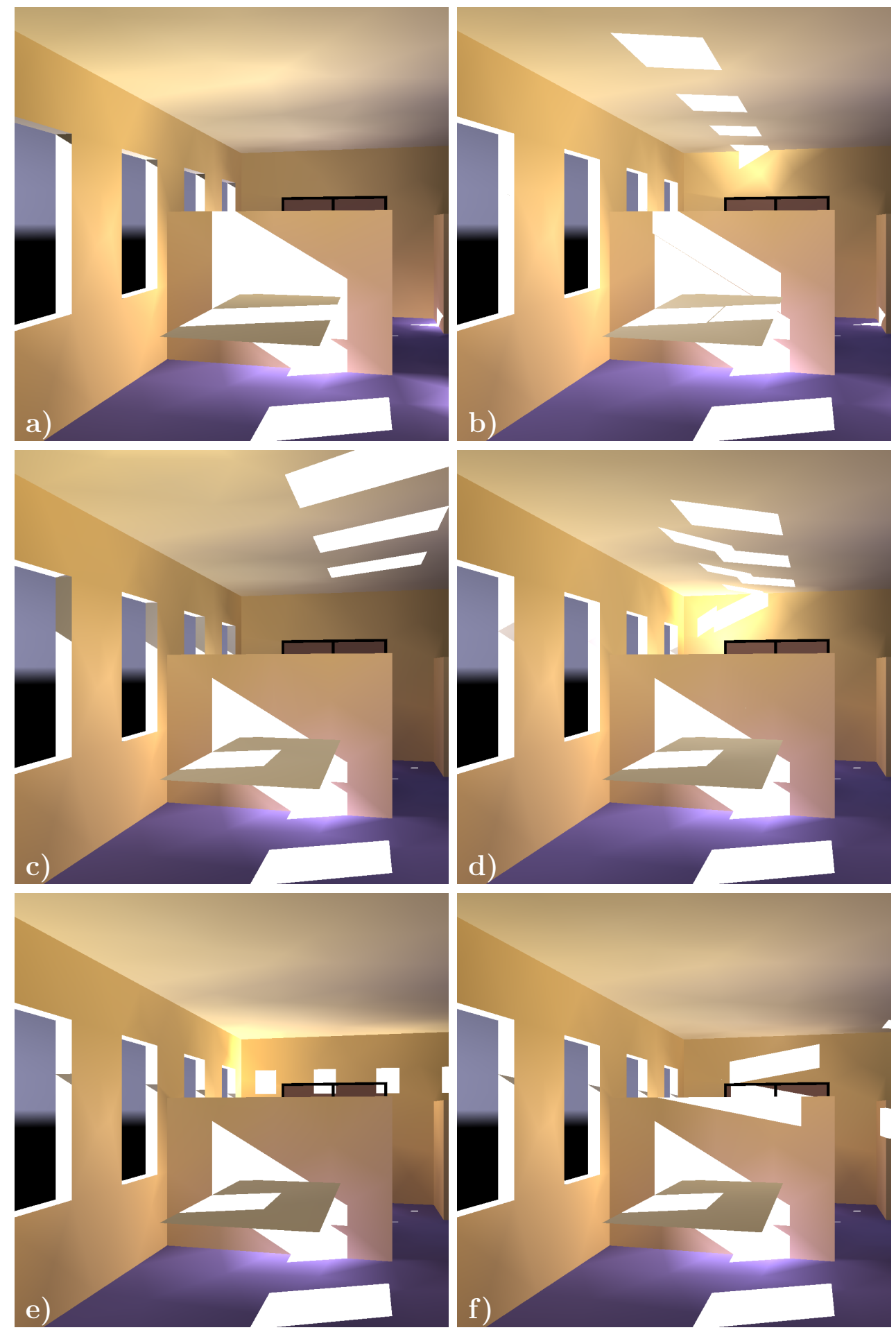

Fig. 8. Sample CFS rendering in a medium-sized office scene with west facing windows at 3:30pm on March 21st. The lower portion of each window is plain glass in all images. The upper portion of each window is a) plain glass, b) laser cut panel, c) mirrored Venetian blind, d) Lumitop ${ }^{\mathrm{TM}}$, e) optical film (interior), and f) Serraglaze $^{\mathrm{TM}}$. 


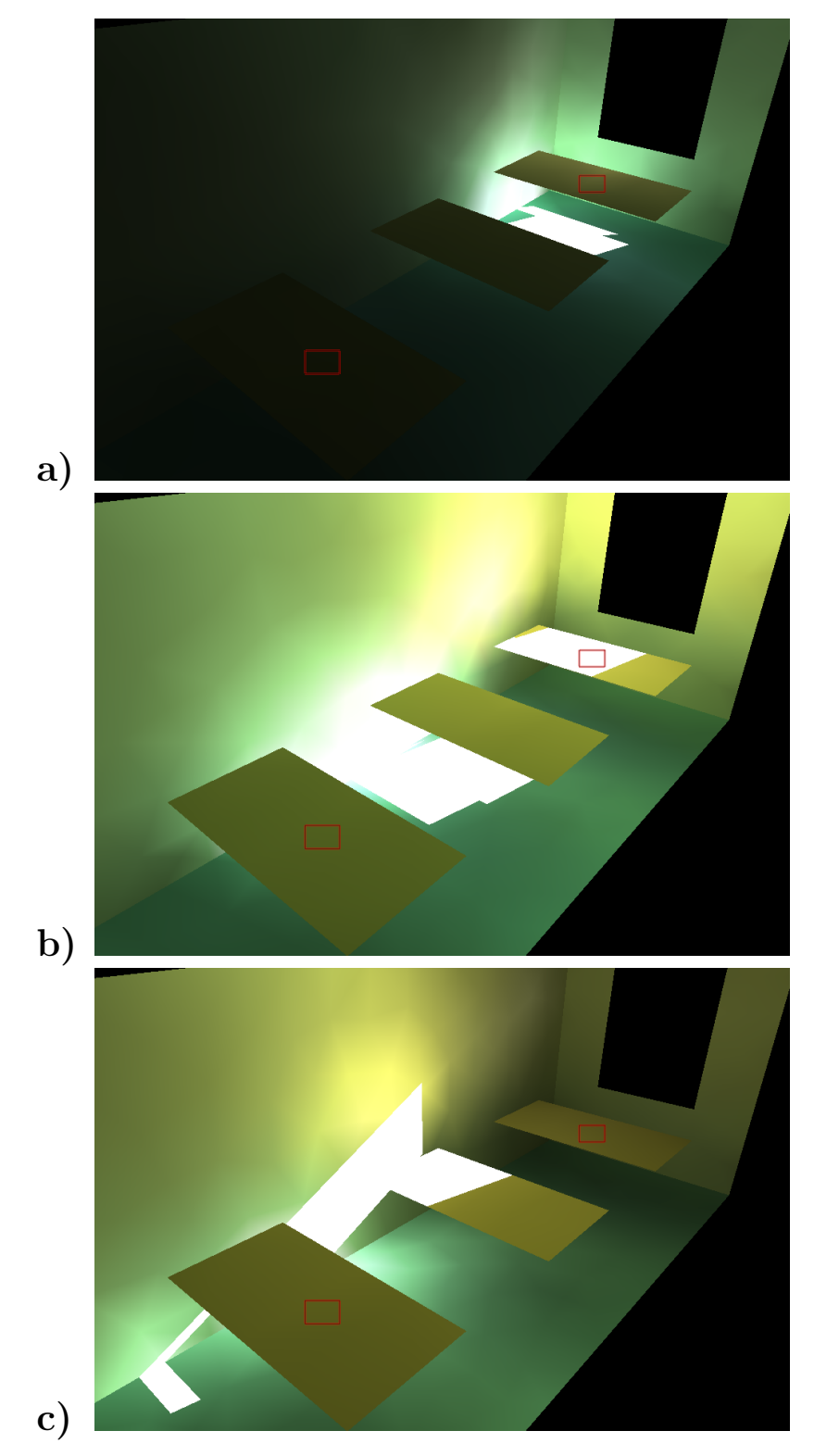

Fig. 9. A simple office scene with a single southern-facing window. The designer uses daylighting optimization to select an appropriate fenestration material to more uniformly illuminate the desks nearest and furthest from the window (outlined with small red squares). The optimization process is shown in Figure 10.

reading locations on the image and the system produces a $2 \mathrm{D}$ plot of the illuminance (lux) on that surface for the free variables. We demonstrate the optimization process for a small office scene with a single window and three desks, shown in Figure 9. The bottom two-thirds of the window is filled with plain glass to preserve the view outside. The designer wishes to select a material for the upper third that maximizes the use of daylight for reading (i.e., minimizing the need for supplemental electric lighting) at the desks throughout the year. 


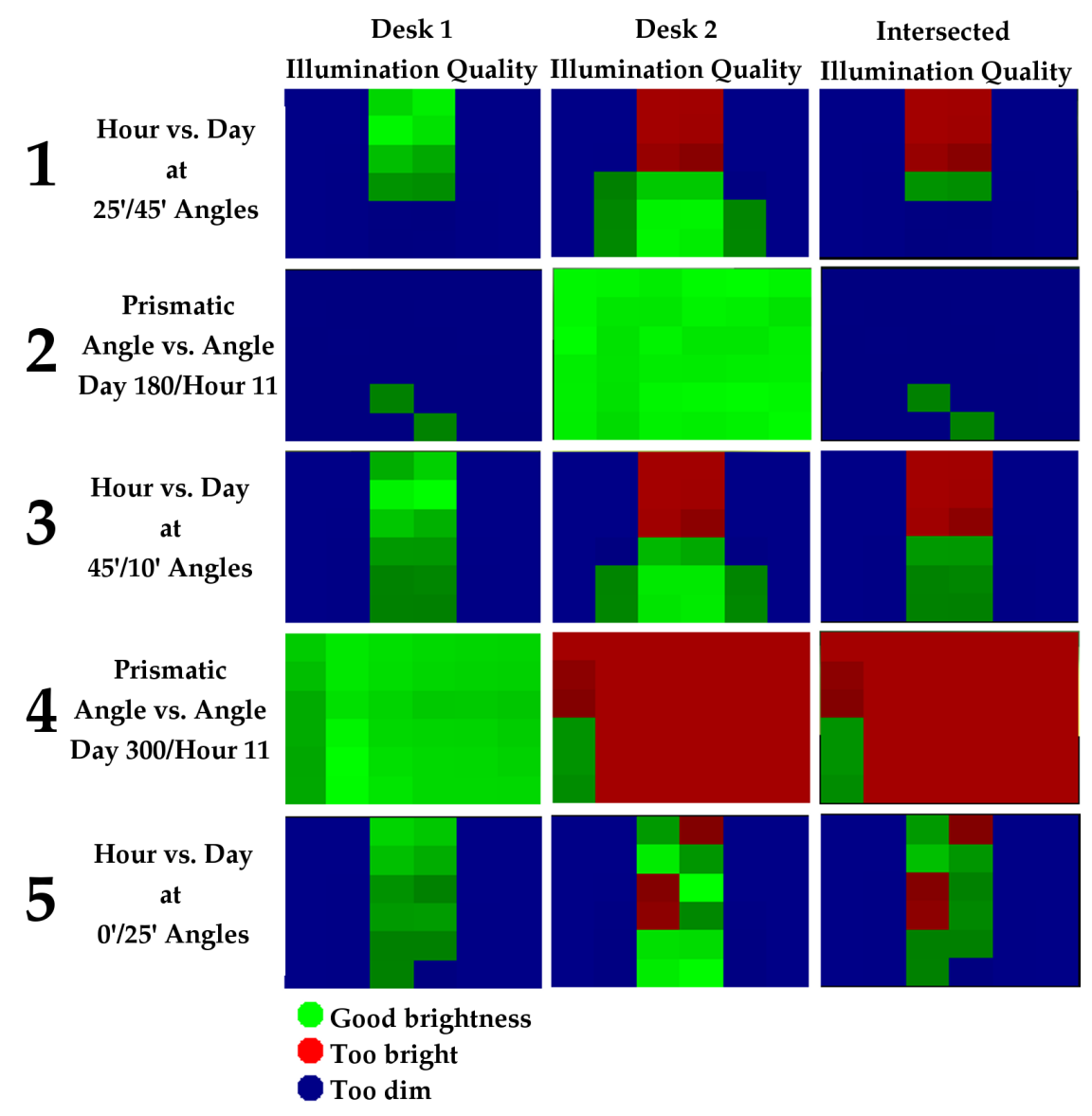

Fig. 10. A multi-stage optimization of the scene shown in Figure 9. The plots are false colored where green indicates levels that fall within the recommended range for reading (200-2000 lux).

Figure 10 illustrates the human-guided optimization process to select the optimal microfacet angles for a prismatic panel to fill the upper third of the window. With an initial choice of angles for the prismatic panel we produce the hour/day plot in Step 1. We see that Desk 1 is never bright enough for reading during the summer (Figure 9a) and when Desk 1 would be bright enough in the winter (Figure $9 \mathrm{~b}$ ) the illumination at Desk 2 is too bright for reading and Desk 2's occupant will probably close the blinds. In Step 2, the designer selects a problematic day and time in the summer and explores the configuration space of microfacet angles. Finding the optimal material selection for summer, the designer returns to the hour/day of year plot in Step 3. The plot has improved, but Desk 2 is still too bright in the winter. Another round of human-guided optimization results in the material selection shown in Step 5 (Figure 9c), which maximizes the daylight autonomy, i.e, the percent of moments in the year when the natural illumination at both desks is appropriate for reading. 


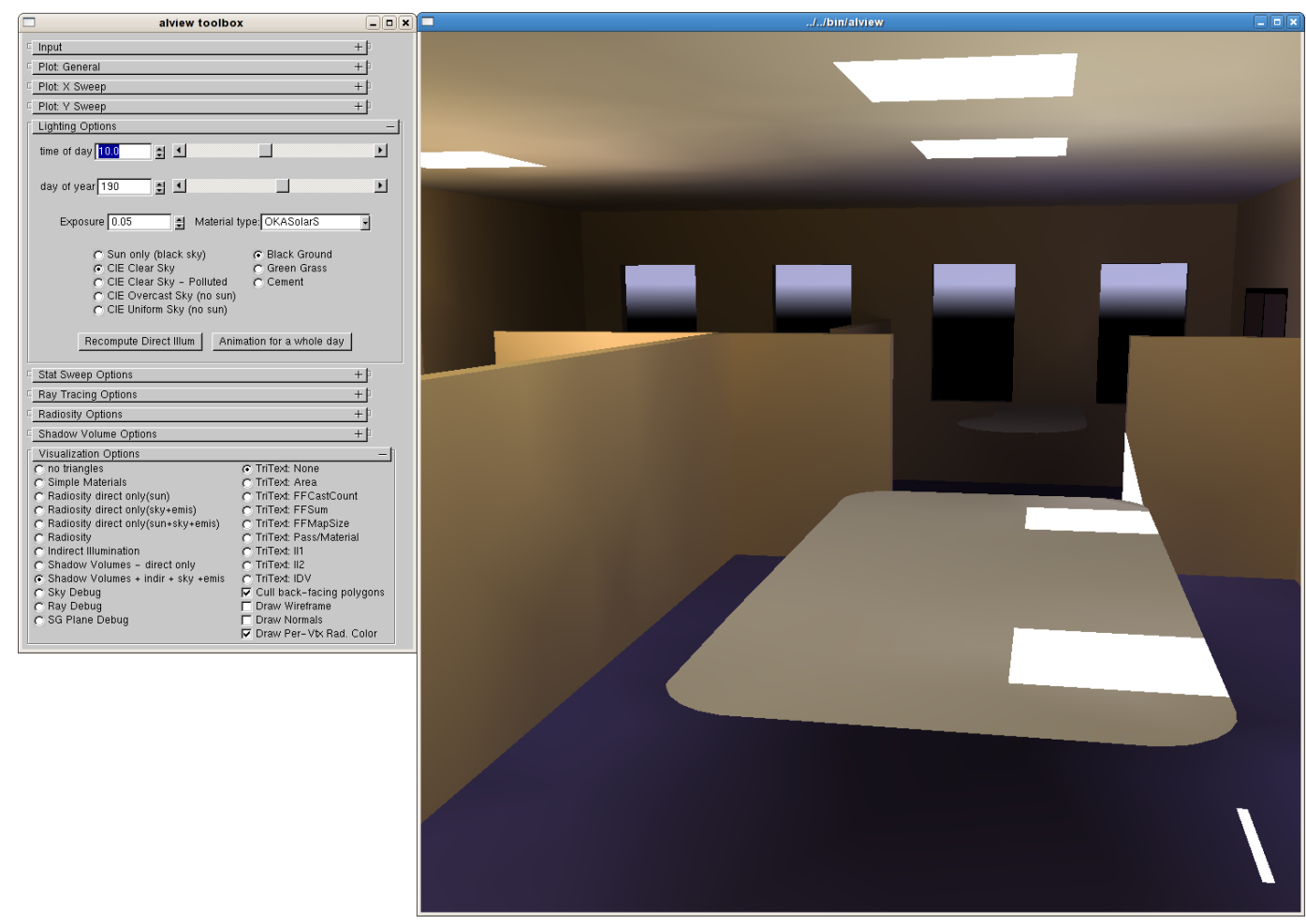

Fig. 11. Our prototype Graphical User Interface (GUI) includes a control panel and visual display window for photorealistic renderings.

\section{Results and Validation}

\subsection{System}

For our interdisciplinary LightSolve architectural daylighting project, we have built an interactive rendering engine based on our hybrid radiosity/shadow volumes rendering method and the CFS modeling and rendering technique. This $\mathrm{C}++$ program runs on Linux, FreeBSD, and Windows/Cygwin with the prototype user interface controls shown in Figure 11. Similar to standard CAD programs, the 3D model can be viewed from arbitrary camera positions with interactive rotation, translation, and zoom. The time of day, day of year, sky conditions (e.g., clear vs. overcast), and different kinds of CFS can be interactively changed. In addition, the renderings can be saved as images and the configuration data stored and used to create corresponding high-accuracy offline Radiance renderings for result validation. 


\subsection{Case Study Example Scenes}

Our results showcase both simple test scenes and more complex real world design situations. The architects we consulted with during the development of our system were quite interested in using daylighting analysis to inform their design processes. Simple test scenes are used in Figures 4, 5, 7, and 9.

Figures 8, 11, and 14 show a moderate-sized office environment with low partition walls and an interesting variety and layout of desk and table furnishings. Few of the desks are close to exterior windows; thus, skylights were used to add illumination to the interior spaces.

The architect of the design shown in Figures 12 and 13 used curvilinear shapes to bring indirect light into a house and enhance the warm colors of late evening sun. The quantitative lux values received by each patch in the scene are not significantly impacted by the low-frequency soft shadow edges inherent in the radiosity method. However, the qualitative visual improvement that the hard shadows in the hybrid radiosity/shadow volume method bring to the results improves the effectiveness of simulation for daylighting design and prevent mistakes such as those seen in Figure 2 and observed by Sweitzer [21].

\subsection{System Validation to Radiance}

For validation of our hybrid radiosity/shadow volumes technique, we compare both the speed and accuracy of our system to Radiance, the accepted industry standard for architectural lighting simulations. We produce two versions of Radiance images for comparison. The first is the ground truth rendering, a highly accurate image that is generated by increasing each of the Radiance parameters until the image is constant. The rendering time to create a single image was 45-90 minutes for our test scenes. Next we adjusted the Radiance parameters to produce a fast rendering, which sacrifices some accuracy in the rendering, but is much quicker (approximately 5 minutes) and thus more useful in schematic architectural design. The comparison was done on a standard PC, with a Intel Core 2 Duo E6400 CPU (2.13GHz), and 2G memory.

\subsubsection{Rendering Speed}

The performance of our prototype implementation is quite compatible with interactive exploration and optimization of CFS for daylighting during the schematic design phase of architecture. A model with 1000-3000 triangular patches is loaded into the system with approximately 10 seconds of initialization to compute the form factors for radiosity. Changing the rotation, transla- 

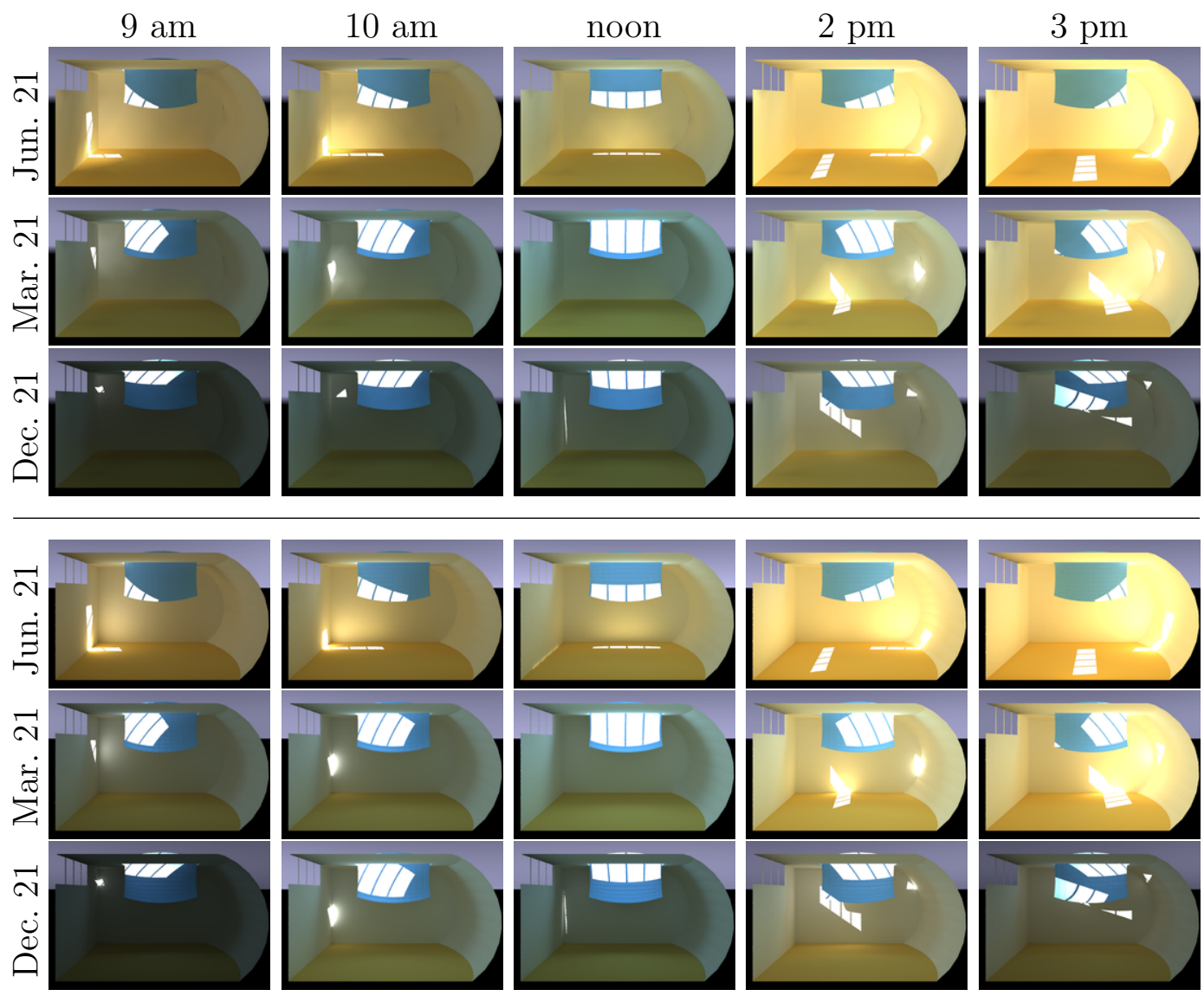

Fig. 12. In designing the interesting curved geometry for the living space in this residential design, the architect redirects the strong overhead noon sunshine from a set of skylights with a curved diffuse blue deflector but allows the warmer late afternoon sun to penetrate deep into the room and wash over the far wall. Our interactive global illumination relighting system allows him to quickly evaluate this geometry for different sun positions and sky conditions. The top 3 rows of images are rendered with our system. The bottom 3 rows are produced by Radiance.

tion, or zoom of the virtual "camera" can be done in realtime ( $>30$ frames per second). When the time of day, day of year, or the parameters of the BTDF for the CFS is changed, the per-pixel direct illumination contribution from the sun is updated in real time using shadow volumes and the contribution from the sky and indirect illumination is progressively updated in approximately 1-3 seconds. Computing an 8x8 plot (e.g., Figure 10) showing many different hour/day or CFS configuration settings thus takes 1-3 minutes.

\subsubsection{Qualitative Comparison of Visual Results}

Figure 12 shows a qualitative comparison of images captured from our system and Radiance at different times and days of the year. Difference images for two of these times are shown in Figure 13 for visual (qualitative) comparison. The Radiance fast rendering (Figure 13a) exhibits locally uneven surface brightness 
artifacts and the total brightness of the scene is incorrect. In contrast, our hybrid radiosity/shadow volumes technique (Figure 13b) matches the ground truth Radiance image (Figure 13c) in both smoothness and overall brightness. From the difference image (Figure 13d), we observe that the primary source of deviation between the renderings is the secondary bounce when bright direct illumination from the sun light falls in the corner of a room. An adaptive meshing method, such as hierarchical radiosity, which we plan implement in future work, will significantly reduce this source of error. Note that errors in the illumination of the curved skylight redirecting panel are due to a lack of surface normals in the Radiance mesh.

\subsubsection{Quantitative Evaluation of Rendering Accuracy}

In lighting design, supplementary fixture selection and placement decisions are made based on predicted numerical illuminance and luminance conditions. Thus we also compare our simulation quantitatively to Radiance, which has been extensively validated for precision use in architectural daylighting design $[53,54,55,56]$. We calculate the luminance difference pixel by pixel between Radiance and our hybrid radiosity/shadow volumes rendering for both the entire image and an area of interest. The results of a quantitative comparison of the luminance values computed in our system and Radiance is shown in Table 1. The margin of error between the systems is less than $10 \%$ for a variety of different scenes, camera positions and daylighting conditions (Figure 14). Thus our system provides accurate qualitative and quantitative renderings appropriate for use in schematic architectural design.

\begin{tabular}{|c|c|c|c|}
\hline Figure & $\begin{array}{l}\text { Radiance average pixel } \\
\text { luminance }\left(\mathrm{cd} / \mathrm{m}^{2}\right)\end{array}$ & $\begin{array}{l}\text { HRSV average pixel } \\
\text { luminance }\left(\mathrm{cd} / \mathrm{m}^{2}\right)\end{array}$ & $\begin{array}{l}\text { average } \\
\text { error }(\%)\end{array}$ \\
\hline \multirow{2}{*}{ 14a) $\begin{array}{l}\text { entire image } \\
\text { desks only }\end{array}$} & 916.40 & 861.35 & 8.2 \\
\hline & 1858.06 & 1748.75 & 7.4 \\
\hline \multirow{2}{*}{$\begin{array}{l}\text { 14b) } \begin{array}{l}\text { entire image } \\
\text { area of interest }\end{array}\end{array}$} & 1414.77 & 1320.76 & 8.6 \\
\hline & 946.51 & 828.62 & 8.8 \\
\hline
\end{tabular}

Table 1

\section{Conclusions and Future Work}

Our rendering speed results compare favorably with the performance data from the study by Glaser et al. [10] of typical use times for the current practice physical heliodon daylighting analysis tool. The architectural models used in that study are of similar complexity to our test cases and our target audience is the same group of users. In that study it took an average of 86 seconds for a user to position the model on the heliodon table. This corresponds to 
a)

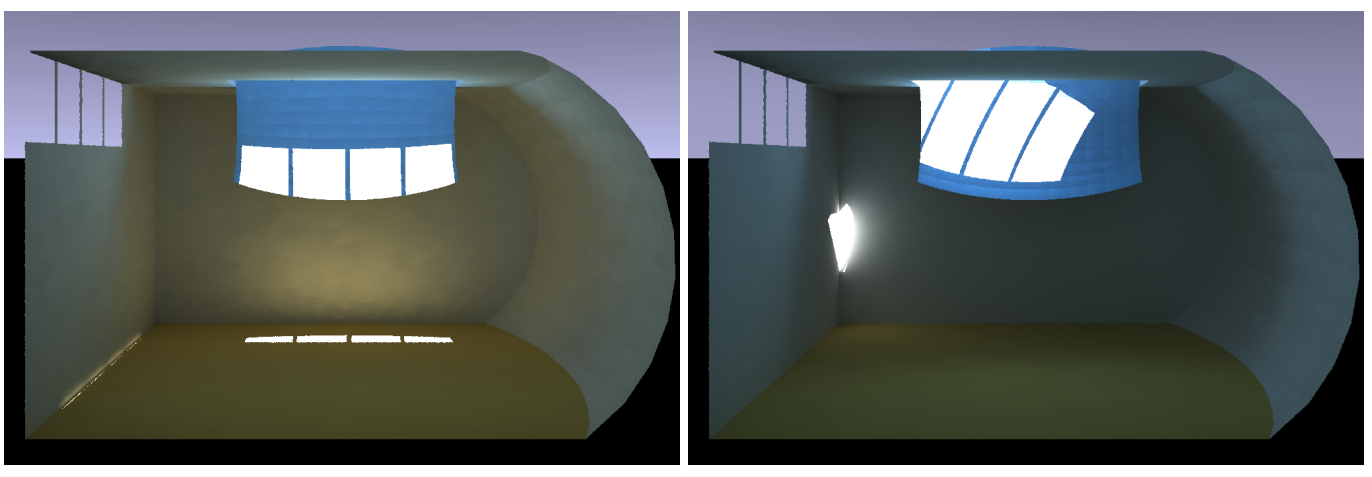

b)

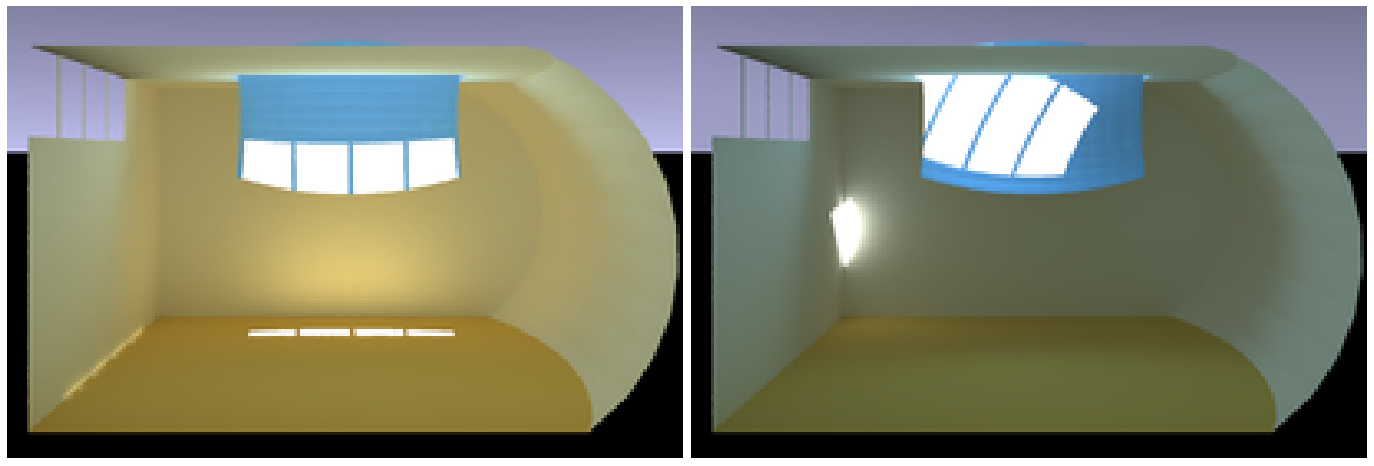

c)

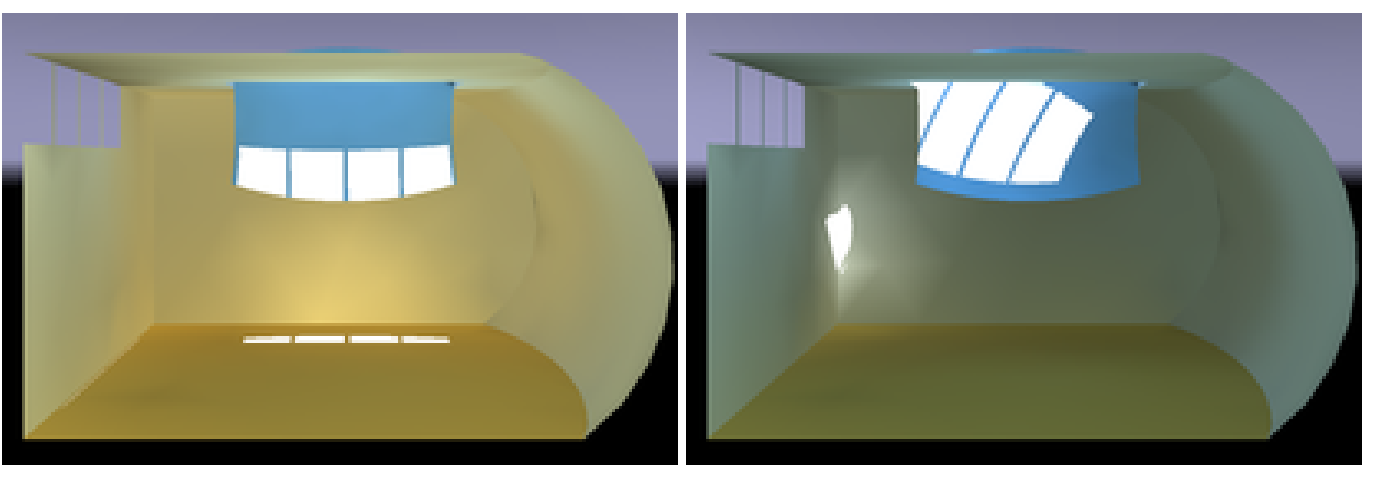

d)

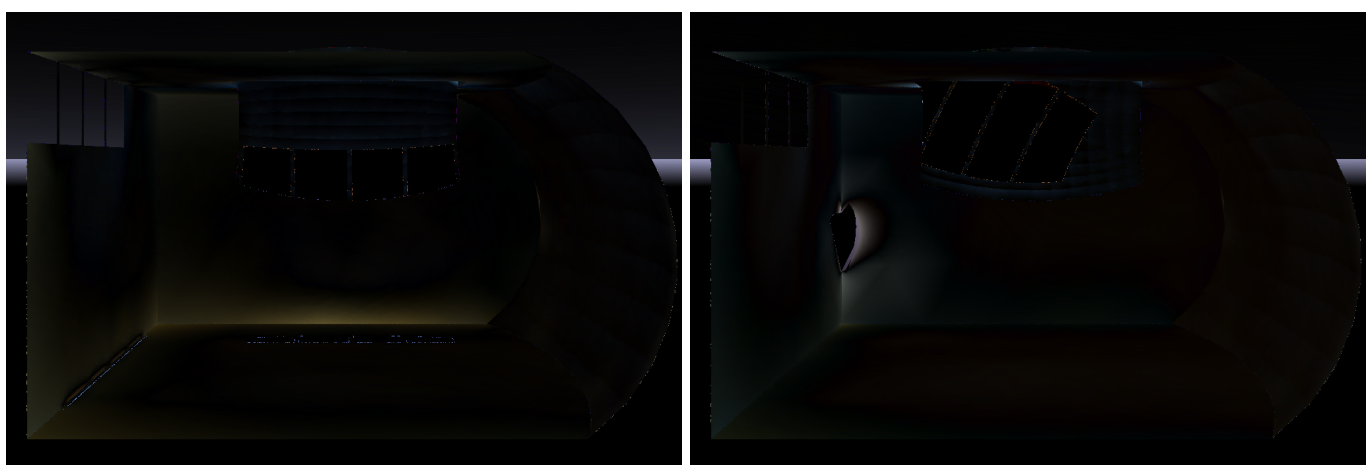

Fig. 13. Qualitatively our interactive renderings are very similar to Radiance's offline renderings: a) Fast Radiance renderings, b) Ground truth Radiance renderings, c) Our hybrid radiosity/shadow volumes method, and d) Difference images between b) and c). 


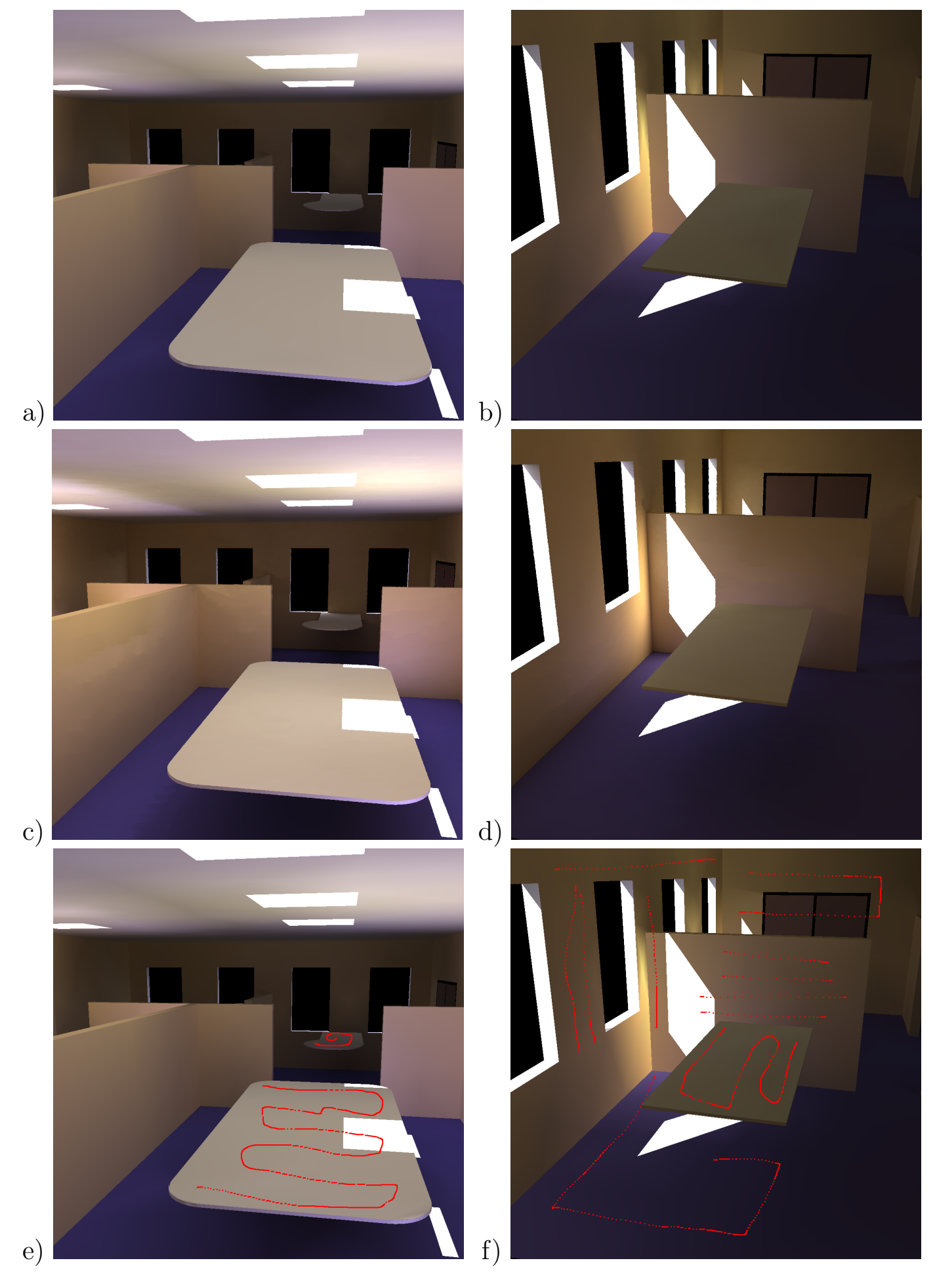

Fig. 14. We performed a quantitative comparison between our system (a \& b) and Radiance (c \& d). The comparisons were done both over all pixels in the image and on a region of interest marked by the user, (shown in red in images e \& f). The numerical results are presented in Table 1. 
our load and initialization time. It took an average of 29 seconds to adjust the heliodon table to capture a new sun position. Likewise, this corresponds to our relighting time. Thus we believe that our tool would integrate well into the typical schematic design phase of architectural design. Furthermore, heliodons model the sun only and can only provide shadow analyses and qualitative studies. Our tool is much more powerful than a heliodon because in addition to qualitative renderings, it includes the sky component, can be used to quantitatively measure light and can model CFS for which physical scale models are not available for use with a heliodon.

Radiance does not support CFS whose transmissive properties are described by BTDF window data. However, this feature is currently under development $[22,57]$ and will include independent validation to certify the accuracy of its prediction of the resulting illumination. We plan to validate our CFS modeling and accelerated rendering to Radiance once this component becomes available.

We are currently working to extend our rendering system to support edits and computer-assisted optimization of the geometry. We have an adaptive form factor sampling framework that facilitates geometry addition, deletion, and modification. Since we are targeting the implementation to architectural students and practitioners, our current implementation makes limited use of advanced graphics hardware. However, we plan to incorporate recent GPU techniques to improve performance as our prototype is expanded. We will also investigate perceptually-appropriate tone mapping of the high dynamic range illumination values throughout the scene. Furthermore, rather than reinvent a CAD modeling user interface, we envision our algorithms as a plug-in to Sketchup [17], a tool that is favored by architects for schematic design. Providing alternate and augmented design tools to architects will improve the energy efficiency and occupant comfort in both new construction and renovation of existing architecture.

\section{Acknowledgments}

The authors were supported by their home institutions for this work. They would like to thank Julie Dorsey, Mark Cabrinha, Magali Bodart, Sian Kleindienst, Tim Herrman, Zachary Cross, and Matthew Ficket.

\section{References}

[1] D. Jenkins and M. Newborough. An approach for estimating the carbon emissions associated with office lighting with a daylight contribution. $A p$ - 
plied Energy, 84:608-622, 2007.

[2] D. Arasteh, J. Apte, and Y.J. Huang. Future Advanced Windows for Zero-Energy Homes. ASHRAE Transactions, 109(2), 2003.

[3] M. Guzowski. Daylighting for Sustainable Design. McGraw-Hill, 2000.

[4] Lisa Heschong. Daylighting and human performance. ASHRAE, June 2002.

[5] A.R. Webb. Considerations for lighting in the built environment: Nonvisual effects of light. Energy and Buildings, 38(7):721-727, 2006.

[6] R. Sullivan, L. Beltran, E.S. Lee, M. Rubin, and S.E. Selkowitz. Energy and Daylight Performance of Angular Selective Glazings. Lawrence Berkeley National Laboratory, Supported by the U.S. Department of Energy, 1998.

[7] M. Kischkoweit-Lopin. An overview of daylighting systems. Solar Energy, $73(2): 7782,2002$.

[8] M. Laar and F.W. Grimme. German developments in guidance systems: an overview. Building Research and Information, 30(4):282-301, July 2002.

[9] H. Köster. Dynamic Daylighting Architecture - Basics, Systems, Projects. Birkhäuser, 2004.

[10] Dan C. Glaser, F. Whitney Smith, and Barbara Cutler. Using video for analyzing daylighting simulation tools. In SimBuild 2006: Building Sustainability and Performance through Simulation, August 2006.

[11] R. Osser, M. Andersen, and L. Norford. Development of Two Heliodon Systems and Recommendations for their Use. In Proceedings of SOLAR 2007: Sustainable Energy Puts America to Work, Cleveland, July 7-12, 2007.

[12] M. Bodart, A. Deneyer, A. De Herde, and P. Wouters. Design of a singlepatch sky and sun simulator. Lighting Research and Technology, 38(1):7389, 2006.

[13] N. Lechner. Heating, Cooling, Lighting. John Wiley \& Sons Inc., 2001.

[14] L. Michel, C. Roecker, and J.-L. Scartezzini. Performance of a new sky scanning simulator. Lighting Research and Technology, 27(4):197-207, 1995.

[15] CIE Commission Internationale de l'Eclairage. Spatial distribution of daylight-cie standard general sky. CIE, S 011/E., 2001.

[16] R. Perez, R. Seals, and J Michalsky. All-weather model for sky luminance distribution - preliminary configuration and validation. Solar Energy, 50(3):235-245, 1993.

[17] SketchUp from Google. SketchUp: 3D modeling software, 2006. http: //www. sketchup.com.

[18] Square One Research. ECOTECT, 2000-2006. www. ecotect.com.

[19] C.F. Reinhart and A. Fitz. Findings from a survey on the current use of daylight simulations during building design. Energy and Buildings, pages 824-835, 2006.

[20] Joseph M. Siry. Carson Pirie Scott: Louis Sullivan and the Chicago De- 
partment Store. The University of Chicago Press, 1956.

[21] G. Sweitzer. Prismatic panel sidelighting systems: Daylighting distribution and electric lighting user patterns in perimeter office workplaces. In Right Light Bright Light, 1991.

[22] M. Andersen and J. de Boer. Goniophotometry and assessment of bidirectional photometric properties of complex fenestration systems. Energy and Buildings, 38(7):836-848, July 2006.

[23] G. Ward Larson and R. Shakespeare. Rendering with Radiance - The Art and Science of Lighting Visualization. Morgan Kaufmann Publishers, Inc., 1998.

[24] Gregory J. Ward. The radiance lighting simulation and rendering system. In Proceedings of SIGGRAPH 94, Computer Graphics Proceedings, Annual Conference Series, pages 459-472, July 1994.

[25] Greg Ward. Radiance: Synthetic imaging system, 1985-1997. http:// radsite.lbl.gov/radiance/HOME.html.

[26] M.A. Lehar and L.R. Glicksman. A simulation tool for predicting the energy implications of advanced facades. In J. Carmeliet, H. Hens, and G.Vermeir, editors, Research In Building Physics, pages 513-518, 2003.

[27] B. Paule, N. Baker, S. Lawton, M. McEvoy, R. Yao, J. de Boer, H. Erhorn, S. Woesner, E. de Groot, L. Zooneveldt, and J.-L. Scartezzini. Dial-europe european daylighting design tool. In Proceedings International Conference on Sustainable Development in Building and Environment, 2003.

[28] C.F. Reinhart, D. Bourgeois, F. Dubrous, A. Laouadi, P. Lopez, and O. Stelescu. Daylight 1-2-3 - a state-of-the-art daylighting/energy analysis software for initial design investigations. In Submitted to Building Simulation 200\%, 2007.

[29] Cindy M. Goral, Kenneth E. Torrance, Donald P. Greenberg, and Bennett Battaile. Modelling the interaction of light between diffuse surfaces. In Computer Graphics (Proceedings of SIGGRAPH 84), volume 18(3), pages 213-222, July 1984.

[30] Michael F. Cohen, Shenchang Eric Chen, John R. Wallace, and Donald P. Greenberg. A progressive refinement approach to fast radiosity image generation. In Computer Graphics (Proceedings of SIGGRAPH 88), volume 22(4), pages 75-84, August 1988.

[31] Shenchang Eric Chen. Incremental radiosity: An extension of progressive radiosity to an interactive image synthesis system. In Computer Graphics (Proceedings of SIGGRAPH 90), volume 24(4), pages 135-144, August 1990.

[32] Alexander Keller. Instant radiosity. In Proceedings of SIGGRAPH 9\%, Computer Graphics Proceedings, Annual Conference Series, pages 49-56, August 1997.

[33] B. Geebelen, M. van der Voorden, and H. Neukermans. Fast and accurate simulation of long-term daylight availability using the radiosity method. Lighting Research and Technology, 37(4):295-312, 2005.

[34] Gregory J. Ward, Francis M. Rubinstein, and Robert D. Clear. A ray 
tracing solution for diffuse interreflection. In SIGGRAPH '88: Proceedings of the 15th annual conference on Computer graphics and interactive techniques, pages 85-92, New York, NY, USA, 1988. ACM Press.

[35] Gregory Ward Larson and Maryann Simmons. The holodeck ray cache: An interactive rendering system for global illumination in non-diffuse environments. ACM Transactions on Graphics, 18(4):361-368, October 1999.

[36] Gene Greger, Peter Shirley, Philip M. Hubbard, and Donald P. Greenberg. The irradiance volume. IEEE Computer Graphics \& Applications, 18(2):32-43, March-April 1998.

[37] Cyrille Damez, Kirill Dmitriev, and Karol Myszkowski. State of the art in global illumination for interactive applications and high-quality animations. Computer Graphics Forum, 22(1):55-77, March 2003.

[38] John R. Wallace, Michael F. Cohen, and Donald P. Greenberg. A twopass solution to the rendering equation: A synthesis of ray tracing and radiosity methods. In Computer Graphics (Proceedings of SIGGRAPH 87), volume 21(4), pages 311-320, July 1987.

[39] Pascal Gautron, Jaroslav Krivánek, Kadi Bouatouch, and Sumanta Pattanaik. Radiance cache splatting: A gpu-friendly global illumination algorithm. In Rendering Techniques 2005: 16th Eurographics Workshop on Rendering, pages 55-64, June 2005.

[40] Carsten Dachsbacher, Marc Stamminger, George Drettakis, and Frédo Durand. Implicit visibility and antiradiance for interactive global illumination. ACM Transactions on Graphics (SIGGRAPH Conference Proceedings), 26(3), August 2007.

[41] Nathan A. Carr, Jesse D. Hall, and John C. Hart. The ray engine. In Graphics Hardware 2002, pages 37-46, September 2002.

[42] Laszlo; Szecsi, Laszlo; Szirmay-Kalos, and Mateu Sbert. Light animation with precomputed light paths on the gpu. In GI '06: Proceedings of the 2006 conference on Graphics interface, pages 187-194, Toronto, Ont., Canada, Canada, 2006. Canadian Information Processing Society.

[43] Greg Coombe, Mark J. Harris, and Anselmo Lastra. Radiosity on graphics hardware. In GI '04: Proceedings of the 2004 conference on Graphics interface, pages 161-168, School of Computer Science, University of Waterloo, Waterloo, Ontario, Canada, 2004. Canadian Human-Computer Communications Society.

[44] Mangesh Nijasure, Sumanta Pattanaik, and Vineet Goel. Interactive global illumination in dynamic environments using commodity graphics hardware. In $P G$ '03: Proceedings of the 11th Pacific Conference on Computer Graphics and Applications, page 450, Washington, DC, USA, 2003. IEEE Computer Society.

[45] J. Marks, B. Andalman, P. A. Beardsley, W. Freeman, S. Gibson, Jessica K. Hodgins, T. Kang, B. Mirtich, H. Pfister, W. Ruml, K. Ryall, J. Seims, and S. Shieber. Design galleries: A general approach to setting parameters for computer graphics and animation. In Proceedings of SIG- 
GRAPH 97, Computer Graphics Proceedings, Annual Conference Series, pages 389-400, August 1997.

[46] David Anderson, Emily Anderson, Neal Lesh, Joe Marks, Ken Perlin, David Ratajczak, and Kathy Ryall. Human-guided simple search: combining information visualization and heuristic search. In Workshop on New Paradigms in Information Visualization and Manipulation, pages 21-25, 1999.

[47] Dani Lischinski, Filippo Tampieri, and Donald P. Greenberg. Combining hierarchical radiosity and discontinuity meshing. In SIGGRAPH '93: Proceedings of the 20th annual conference on Computer graphics and interactive techniques, pages 199-208, New York, NY, USA, 1993. ACM.

[48] Franklin C. Crow. Shadow algorithms for computer graphics. In Computer Graphics (Proceedings of SIGGRAPH 77), volume 11(2), pages 242248, July 1977.

[49] Tim Heidmann. Real shadows, real time. Iris Universe, 18:28-31, 1991. Silicon Graphics, Inc.

[50] Morgan McGuire, John F. Hughes, Kevin Egan, Mark Kilgard, and Cass Everitt. Fast, practical and robust shadows. Technical report, NVIDIA Corporation, Austin, TX, Nov 2003. http://developer.nvidia.com/ object/fast_shadow_volumes.html.

[51] Andrew Woo, Pierre Poulin, and Alain Fournier. A survey of shadow algorithms. IEEE Comput. Graph. Appl., 10(6):13-32, 1990.

[52] M. Andersen, C. Roecker, and J.-L. Scartezzini. Design of a time-efficient video-goniophotometer combining bidirectional functions assessment in transmission and reflection. Solar Energy Materials and Solar Cells, 88(1):97-118, June 2005.

[53] J. Mardaljevic. Validation of a lighting simulation program under real sky conditions. Lighting Research and Technology, 27(4):181-188, 1995.

[54] John Mardaljevic. The bre-idmp dataset: A new benchmark for the validation of illuminance prediction techniques. Lighting Research and Technology, 33(2):117-136, 2001.

[55] J. Mardaljevic. Verification of program accuracy for illuminance modelling: assumptions, methodology and an examination of conflicting findings. Lighting Research and Technology, 36(3):217-242, 2004.

[56] Anca D. Galasiu and Morad R. Atif. Applicability of daylighting computer modeling in real case studies: Comparison between measured and simulated daylight availability and lighting consumption. Building and Environment, 37:363-377, 2002.

[57] Greg Ward. Utilizing BTDF Window Data, 2007. "6th International Radiance and HDR Scientific Workshop". 